H. SHIGA AND H. TANIGAWA

KODAI MATH. J.

12 (1989), 437-443

\title{
ON THE MASKIT COORDINATES OF TEICHMÜLLER SPACES AND MODULAR TRANSFORMATIONS
}

\author{
By Hiroshige Shiga and Harumi Tanigawa
}

\section{$\S 1$. Introduction}

Let $S$ be a Riemann surface of type $(p, n)$ with $3 p-3+n>0$. As is wellknown, the Teichmüller space $T(S)$ of $S$ is a $3 p-3+n$ dimensional complex manifold. Maskit [9] has observed that the deformation space $T(\Gamma)$ of some kind of Kleinian group $\Gamma$ is a model of $T(S)$, and he also has described an embedding $T(S) \rightarrow U^{3 p-3+n}$, where $U$ is the upper half plane. The group $\Gamma$ is defined by choosing an admissible set of $3 p-3+n$ curves $\Sigma$ on $S$. The embedding does not depend on the base point of $T(S)$ and depends only on the choice of curves, which is done in a finitely many ways. The embedding yields global coordinates of $T(S)$, called the Maskit coordinates, and with these coordinates the modular transformation induced by a Dehn twist about an element of $\Sigma$ is represented by a parabolic element of $\operatorname{PSL}(2, \boldsymbol{R})$.

In this paper we shall investigate some properties of Maskit coordinates. In $\S 4$, we shall observe a certain type of modular transformations. In $\S 5$, we shall give a sufficient condition for parabolic modular transformations to have an invariant Teichmüller disc.

\section{§ 2. Preliminaries}

In this section we recall some known results and fix our notations. Let $S$ be a Riemann surface of type $(p, n)$ with $3 p-3+n>0$, and let $f$ and $g$ be quasiconformal mappings of $S$ onto $S^{\prime}$ and $S^{\prime \prime}$, respectively. The mappings $f$ and $g$ are said to be equivalent if there exists a conformal mapping $h$ of $S^{\prime}$ onto $S^{\prime \prime}$ such that $g^{-1} \circ h \circ f: S \rightarrow S$ is homotopic to the identity. The Teichmüller space $T(S)$ of $S$ is the set of all these equivalence classes. It is known that $T(S)$ is a $3 p-3+n$ dimensional complex manifold.

$T(S)$ is a metric space with the Teichmüller distance

$$
d(q, p)=\frac{1}{2} \inf \left\{\log K_{f \circ g^{-1}} \mid f \in p, g \in q\right\}
$$

where $K_{f \circ g^{-1}}$ denotes the maximal dilatation of $g \circ f^{-1}$. The Teichmüller distance

Received February 10, 1989; Revised April 25, 1989. 
coincides with the Kobayashi distance on $T(S)$. The image of an holomorphic isometry of the upper half plane $U$ into $T(S)$ with respect to the hyperbolic distance on $U$ and the Teichmüller distance on $T(S)$ is called a Teichmuller disc.

Let $\omega$ be a quasiconformal self-mapping of $S$. The self-mapping of $T(S)$ of the form $\chi[\omega]:[f] \rightarrow\left[f \circ \omega^{-1}\right]$ is biholomorphic and is isometric with respect to the Teichmüller distanse on $T(S)$. Such an automorphism of $T(S)$ is called a modular transformation of $T(S)$. The set of all modular transformations is called the modular group of $T(S)$, and is denoted by $\operatorname{Mod}(S)$.

A non-empty set of disjoint closed Jordan curves $C=\left\{C_{1}, \cdots, C_{l}\right\}$ on $S$ is called admissible if no $C_{j}$ is homotopic to a point, a puncture or $C_{k}(k \neq j)$. An orientation preserving homeomorphism $f: S \rightarrow S$ is called to be reduced by $C$ if $f(C)=C$. A self-mapping of $S$ is called reducible if it is homotopic to a reduced mappings, and is called irreducible otherwise. Let $f$ be reduced by $C$. For each component $S^{(k)}$ of $S-C$, there is the smallest positive integer $J(k)$ such that $f^{J(k)}\left(S^{(k)}\right)=S^{(k)}$. If $f^{J(k)} \mid S^{(k)}$ is irreducible as a self-mapping of $S^{(k)}$ for each $S^{(k)}$, then $f$ is called to be completely reduced by $C$. Bers [2] showed that every reducible self-mapping is homotopic to a completely reduced mapping.

Let $\chi$ be an element of $\operatorname{Mod}(S)$ and not the identity. We shall say that $\chi$ is elliptic if it is periodic, and non-periodic $\chi$ is hyperbolic if it is induced by an irreducible mapping, parabolic if there exists a reduced mapping $f$ by some admissible set of curves $C$ such that $f$ induces $\chi$ and for each part $S^{(k)}$ of $S-C$ $f^{J(k)} \mid S^{(k)}$ is homotopic to a periodic selfmapping of $S^{(k)}$, and pseudo-hyperbolic otherwise (cf. Bers [2]). For each element $\chi$ of $\operatorname{Mod}(S)$, set $a(\chi)=\inf \{d(p, \chi(p))$; $p \in T(S)\}$. It is known (Bers [2]) that $\chi$ is elliptic if and only if $\chi$ has a fixed point, parabolic if and only if $a(\chi)=0$ and there is no fixed point, hyperbolic if and only if $a(\chi)>0$ and there is a $p \in T(S)$ with $a(\chi)=d(p, \chi(p))$, pseudo-hyperbolic if and only if $a(\chi)>0$ and no point of $T(S)$ attains $a(\chi)$.

A finitely generated non-elementary Kleinian group $G$ is called to be a $b$ group if it has exactly one simply connected invariant component $\Delta(G)$ of the region of discontinuity $\Omega(G)$. If there is a parabolic element of a $b$-group $G$ not corresponding to a puncture of $\Delta(G) / G$, then it is called an accidental parabolic transformation. We call a $b$-group $G$ to be regular if the Poincaré area of $\Delta(G) / G$ is half of that of $\Omega(G) / G$ (cf. Bers [1], Maskit [8]).

For an arbitrary Kleinian group $G$, a quasiconformal self-mapping $w$ of $\hat{\boldsymbol{C}}$ is G-compatible if $w \circ \gamma \circ w^{-1} \in P S L(2, \boldsymbol{C})$ for all $\gamma \in G$. Two $G$-compatible quasiconformal mapping $w_{1}$ and $w_{2}$ are said to be equivalent if there exists an $A \in$ $P S L(2, C)$ such that $w_{1} \circ \gamma \circ w_{1}^{-1}=A \circ w_{2} \circ \gamma \circ w_{2}^{-1} \circ A^{-1}$ for all $\gamma \in G$. The deformation space $T(G)$ is the set of all equivalence classes $[w]_{G}$ of $G$-compatible quasiconformal mappings $w$.

\section{§ 3. Maskit coordinates of Teichmüller spaces}

Now we shall introduce the embedding $T(S) \rightarrow U^{3 p-3+n}$, where $U$ is the upper half plane, due to Maskit [9] and Kra [6]. 
An admissible set of $3 p-3+n$ Jordan curves $\Sigma=\left\{a_{1}, \cdots, a_{3 p-3+n}\right\}$ on $S$ is called a maximal partition of $S$. It is known (Maskit [8]) that there exists a torsion free regular $b$-group $\Gamma$ satisfying:

1) $(\Omega(\Gamma)-\Delta(\Gamma)) / \Gamma$ is a union of $2 p-2+n$ thrice punctured spheres,

2) $\Delta(\Gamma) / \Gamma$ is conformally equivalent to $S$,

3) there is a one to one correspondence between conjugacy classes of primitive accidental parabolic elements of $\Gamma$ and $\left\{a_{1}, \cdots, a_{3 p-3+n}\right\}$.

It is also known that $\Gamma$ is determined by $(S, \Sigma)$ uniquely up to conjugation in $P S L(2, \boldsymbol{C})$. We say that $\Gamma$ represents $(S, \Sigma)$. A regular $b$-group with the property 1 ) is called terminal. For a terminal $b$-group $G$, it is known that $T(G)$ is canonically identified with $T(\Delta(G) / G)$. We shall make no distinction between them.

$S-\Sigma$ is a union of $2 p-2+n$ connected components: $S-\Sigma=S_{1} \cup \cdots \cup S_{2 p-2+n}$. Each component $S_{\imath}$ is topologically equivalent to a thrice punctured sphere. Let $\pi: \Delta(\Gamma) \rightarrow \Delta(\Gamma) / \Gamma$ be the canonical projection. The stabilizer of each component of $\pi^{-1}\left(S_{2}\right)$ in $\Gamma$ is a triangle group $(i=1, \cdots, 2 p-2+n)$. Here, we consider a decomposition of $\Gamma$. Let $S^{\prime}$ be a region of $S$ whose boundary is a union of elements of $\Sigma$ and punctures of $S$. The stabilizer $\Gamma^{\prime}$ of each component of $\pi^{-1}\left(S^{\prime}\right)$ in $\Gamma$ is a terminal regular $b$-group, we may assume that it is constructed from some of these triangle groups taken as above by means of two types of operations (Kra [6]):

1) amalgamated free products across cyclic groups, and

2) HNN extensions obtained by conjugating, via a loxodromic element of

$\Gamma$, one cyclic parabolic group into another,

It is also known that $\Gamma^{\prime}$ represents $\left(\Delta\left(\Gamma^{\prime}\right) / \Gamma^{\prime}, \Sigma \cap S^{\prime}\right)$ and that $\Delta(\Gamma) / \Gamma^{\prime}$ is topologically equivalent to $S^{\prime}$.

We shall denote by $T_{\imath}$ the component of $S-\left(\Sigma-\left\{a_{\imath}\right\}\right)$ containing $\left\{a_{\imath}\right\}$ (i= $1, \cdots, 3 p-3+n)$, and also denote by $G_{i}$ the stabilizer in $\Gamma$ of a component of $\pi^{-1}\left(T_{\imath}\right)$. Since $\Sigma$ is a maximal partition, $T_{\imath}$ is a surface of type $(1,1)$ or $(0,4)$ and $\left\{a_{1}\right\}$ is a maximal partition of $T_{2} . \Delta\left(G_{i}\right) / G_{i}$ is topologically equivalent to $T_{\imath}$.

We proceed to introduce the embedding of $T(\Gamma)(=T(S))$. For $i=1, \cdots, n$, every $\Gamma$-compatible quasiconformal mapping is $G_{i}$-compatible. Thus there is a natural map $m: T(\Gamma) \rightarrow \Pi_{j=1}^{3 p-3+n} T\left(G_{j}\right)$, defined by:

$$
m\left([w]_{\Gamma}\right)=\left([w]_{G_{1}}, \cdots,[w]_{G_{3 p-3+n}}\right) .
$$

The mapping $m$ is known to be holomorphic injection onto an open subset of $\Pi_{j=1}^{3 p-3+n} T\left(G_{j}\right)$ (Maskit [9], Kra [6]). Each $T\left(G_{i}\right)$ is canonically identified with the upper half plane $U$. This identification depends only on the conjugacy class of $G_{i}$ in $P S L(2, C)$. We call the representation of $T(\Gamma)$ via $m$ to $U^{3 p-3+n}$ Maskit coordinates of $T(\Gamma)$ (Kra [6]). We shall identify $T(S)$ and $m(T(S)$ ), and regard it as a domain of $U^{3 p-3+n}$.

Under this identification, it is easily seen that the modular transformation induced by a Dehn twist about $a$, is of the form (Maskit [8]): 


$$
\begin{aligned}
& \left(z_{1}, \cdots, z_{\jmath-1}, z_{\jmath}, z_{\jmath+1}, \cdots, z_{3 p+3+n}\right) \\
& \longrightarrow\left(z_{1}, \cdots, z_{\jmath-1}, z_{j}+1, z_{j+1}, \cdots, z_{3 p-3+n}\right) \quad(j=1, \cdots, 3 p-3+n) .
\end{aligned}
$$

\section{$\S 4$. Some properties of Maskit coordinates}

In this section, we shall investigate Maskit coordinates of Teichmüller spaces and a certain type of modular transformations.

Let $S, \Sigma, \Gamma$, and $\left\{T_{2}\right\}_{i=1}^{3 p+n}$ be the same ones as in the preceding section. We assume that the open subset $S^{\prime}=T_{1} \cup \cdots \cup T_{l}$ is connected. Recall that $\Gamma^{\prime}$, the stabilizer of a component of $\pi^{-1}\left(S^{\prime}\right)$ in $\Gamma$ is a terminal $b$-group representing $\left(\Delta\left(\Gamma^{\prime}\right) / \Gamma^{\prime}, S^{\prime} \cap \Sigma\right)$.

LEMMA 1. The projection $\pi_{1 \ldots l}:\left(z_{1}, \cdots, z_{3 p-3+n}\right) \rightarrow\left(z_{1}, \cdots, z_{l}\right)\left(\left(z_{1}, \cdots, z_{3 p-3+n}\right)\right.$ $\in T(S))$ is a surjective mapping of $T(S)$ onto $T\left(\Delta\left(\Gamma^{\prime}\right) / \Gamma^{\prime}\right)$.

Proof. (See also Kra [5]) Each $\left(z_{1}, \cdots, z_{l}\right) \in T\left(\Delta\left(\Gamma^{\prime}\right) / \Gamma^{\prime}\right)$ corresponds to a terminal regular $b$-group $\tilde{\Gamma}^{\prime}$ being quasiconformaly equivalent to $\Gamma^{\prime}$. By the argument as in the proof of Maskit [7] Theorem 5, 6, the following fact is shown. By means of amalgamated free products and $H N N$ extentions, we can construct from $\tilde{\Gamma}^{\prime}$ a terminal regular $b$-group $\tilde{\Gamma}$ which is a quasiconformal deformation of $\Gamma$, and corresponds to a point $p$ of $T(\Gamma)$ such that $\pi_{1 \ldots l}(p)=$ $\left(z_{1}, \cdots, z_{l}\right)$. Thus we have $\pi_{1 \cdots l}(T(S)) \supset T\left(\Delta\left(\Gamma^{\prime}\right) / \Gamma^{\prime}\right.$. Conversely, each $\left(z_{1}, \cdots\right.$, $\left.z_{3 p-3+n}\right) \in T(\Gamma)$ corresponds to a $\Gamma$-compatible quasiconformal mapping $w$. Considering $w$ as a $\Gamma^{\prime}$-compatible quasiconformal mapping, we see that the equivalence class of $w$ corresponds to $\left(z_{1}, \cdots, z_{l}\right)$. Hence $\pi_{1 \ldots l}(T(S)) \subset T\left(\Delta\left(\Gamma^{\prime}\right) / \Gamma^{\prime}\right.$,

THEOREM 1. Let $\chi[w]$ be a modular transformation induced by a quasiconformal mapping $w$ of $S$ which is completely reduced by $\Sigma$. Then there exist $3 p-3+n$ elliptic or parabolic elements of $\operatorname{PSL}(2, \boldsymbol{R}) f_{1}, \cdots, f_{3 p-3+n}$ and an element $\sigma$ of $\mathfrak{S}_{3 p-3+n}$, such that

$$
\begin{gathered}
\chi[w]\left(z_{1}, \cdots, z_{3 p-3+n}\right)=\left(f_{1}\left(z_{\sigma(1)}\right), \cdots, f_{3 p-3+n}\left(z_{\sigma(3 p-3+n)}\right)\right) \\
\left(z_{1}, \cdots, z_{3 p-3+n}\right) \in T(S),
\end{gathered}
$$

where $\mathfrak{S}_{3 p-3+n}$ is the permutation group of degree $3 p-3+n$.

Proof. Set $\chi[w](z)=\left(\Phi_{1}(z), \cdots, \Phi_{3 p-3+n}(z)\right), z \in T(S)$. Since $w(\Sigma)=\Sigma$, the Kleinian group $\Gamma$ representing $(S, \Sigma)$ also represents $\left(S, w^{-1}(\Sigma)\right)$, and there is an element $\sigma$ of $\widetilde{S}_{3 p-3+n}$ such that $w^{-1}\left(T_{\imath}\right)=T_{\sigma(i)}(i=1, \cdots, 3 p-3+n)$. We shall show that each $\Phi_{i}(z)$ depends only on $z_{\sigma(i)}$. Let $\tilde{w}$ be a lift of $w$ on $\Delta(\Gamma)$, so that the following diagram is commutative.

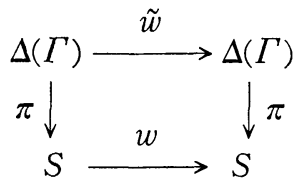


Here, $\pi$ is the natural projection of $\Delta(\Gamma)$ onto $S=\Delta(\Gamma) / \Gamma$. Then there exists an isomorphism $\rho$ of $\Gamma$ to itself such that $\tilde{w} \circ \gamma=\rho(\gamma) \circ \tilde{w}$. Since $\tilde{w}^{-1}$ maps each component of $\pi^{-1}\left(T_{2}\right)$ onto a component of $\pi^{-1}\left(T_{\sigma(i)}\right), \rho\left(G_{i}\right)$ is the stabilizer in $\Gamma$ of a component of $\pi^{-1}\left(T_{\sigma(i)}\right)$, where $G_{i}$ is the stabilizer in $\Gamma$ of a component of $\pi^{-1}\left(T_{\imath}\right) . \quad \Phi_{\imath}$ is determined by $\rho\left(G_{i}\right)$, hence $\Phi_{\imath}$ depends only on $G_{\sigma(i)}$, the terminal $b$-group determining $z_{\sigma(i)}$. Thus we see that $\Phi_{i}(z)=f\left(z_{\sigma(i)}\right)$ is a holomorphic mapping of $z_{\sigma(i)}$. From Lemma 1, both of the domain and the range of $f_{\imath}$ are $U$. By the same argument as above, $\chi[w]^{-1}(z)=\chi\left[w^{-1}\right](z)=\left(g_{1}\left(z_{\tau(i)}\right)\right.$, $\left.\cdots, g_{3 p-3+n}\left(z_{\tau(3 p-3+n)}\right)\right)$, where $\tau=\sigma^{-1} \in \widetilde{S}_{3 p-3+n}$ and each $g_{2}$ is a holomorphic mapping of $U$ onto $U$. Since $g_{i} \circ f_{\tau(i)}\left(z_{\imath}\right)=z_{\imath}\left(z_{i} \in U\right), f_{\imath}$ is injective. Hence $f_{i} \in P S L(2, \boldsymbol{R})$.

Since $\rho\left(G_{i}\right)$ and $G_{\sigma(i)}$ are conjugate in $\Gamma$, we may assume $\rho\left(G_{\imath}\right)=G_{\sigma(i)}$. Since $\Delta\left(\rho\left(G_{i}\right)\right) / \rho\left(G_{i}\right)=\Delta\left(G_{\sigma(i)}\right) / G_{\sigma(i)}$, the transformation $z_{\sigma(i)} \rightarrow f_{i}\left(z_{\sigma(i)}\right)$ is a modular transformation of $T\left(\Delta\left(G_{\sigma(i)}\right) / G_{\sigma(i)}\right)$ induced by a selfmapping $w_{\sigma(i)}$ of $\Delta\left(G_{\sigma(i)}\right) /$ $G_{\sigma(i)}$. In other words, $f_{\imath}$ is represented as following;

$$
f_{\imath}:[f] \longrightarrow\left[f \circ w_{\sigma(2)}^{-1}\right], \quad[f] \in T\left(\Delta\left(G_{\sigma(i)}\right) / G_{\sigma(i)}\right)
$$

$[i d] \in T\left(\Delta\left(G_{\sigma(i)}\right) / G_{\sigma(i)}\right)$ corresponds to a terminal regular $b$-group representing $\left(\Delta\left(G_{\sigma(i)}\right), a_{\sigma(i)}\right)$, and $\left[w_{\sigma(i)}^{-1}\right] \in T\left(G_{\sigma(i)} / G_{\sigma(i)}\right)$ corresponds to a terminal regular $b$-group representing $\left(\Delta\left(G_{\sigma(i)}\right), w_{\sigma(i)}^{-1}\left(a_{\sigma(i)}\right)\right)$. On the other hand, since $w^{-1}\left(a_{\imath}\right)=$ $a_{\sigma(i)}, f_{i}([i d])$ corresponds to the terminal regular $b$-group representing $\left(\Delta\left(\rho\left(G_{i}\right)\right)\right.$ $\left./ \rho\left(G_{i}\right), a_{\sigma(i)}\right)$. Since $f_{i}([i d])=\left[w_{\sigma(i)}^{-1}\right], w_{\sigma(i)}^{-1}\left(a_{\sigma(i)}\right)$ and $a_{\sigma(i)}$ are in the same homotopy class. Hence $w_{\sigma(i)}$ is reducible. This means that $f_{\imath}$ is not hyperbolic modular transformation of $T\left(\Delta\left(G_{\sigma(i)}\right) / G_{\sigma(i)}\right)$. Under the identification of $T\left(\Delta\left(G_{\sigma(i)}\right)\right.$ $\left./ G_{\sigma(i)}\right)$ with $U$, the Teichmüller metric is the hyperbolic metric. Hence $f_{\imath}$ is a non-hyperbolic element of $P S L(2, \boldsymbol{R})$ (cf. Kra [4]).

It is known (Royden [10]) that if $\operatorname{dim} T(S) \geqq 2 \operatorname{Mod}(S)$ is the group of holomorphic automorphisms of $T(S)$, and acts properly discontinuously on $T(S)$. Since there exist uncountably many conformal automorphisms of $U^{3 p-3+n}, T(S)$ $\neq U^{3 p-3+n}$. Furthermore the following fact is shown.

CoRollary (to Lemma 1). With respect to Maskit coordinates, $T(S)$ is not represented as following;

$$
T(S)=U_{1} \times U_{2}
$$

where $U_{1}\left(\right.$ resp. $\left.U_{2}\right)$ is an open subset of $U^{k}$ (resp. $U^{3 p-3+n-k}$ ) corresponding to the first $k$ coordinates (resp. the last $3 p-3+n-k$ coordinates). Here, $k$ is a positive integer.

Proof. The proof is given by induction on $\operatorname{dim} T(S)=3 p-3+n$. For $3 p-3+n=2$, if $T(S)$ was represented as the direct product of $U_{1}$ and $U_{2}$, then from Lemma 1 we see that $U_{1}$ and $U_{2}$ coincide with $U$. This contradicts the proper discontinuity of the action of $\operatorname{Mod}(S)$. Suppose that the statement 
holds for the case in which $\operatorname{dim} T(S) \leqq m-1$, and that $T(S)=U_{1} \times U_{2}$, where $U_{1}$ and $U_{2}$ are open subsets of $U^{k}$ and $U^{m-k}$ respectively as in the statement of the corollary. If $k=1$, then $U_{1}=U$ from Lemma 1 . Thus, all transformations of the form: $\left(z_{1}, z_{2}, \cdots, z_{m}\right) \rightarrow\left(f\left(z_{1}\right), z_{2}, \cdots, z_{m}\right), f \in P S L(2, \boldsymbol{R})$ are contained in $\operatorname{Mod}(S)$. This contradicts the discontinuity of the action of $\operatorname{Mod}(S)$. Hence $k \geqq 2$, and by the same reason $m-k \geqq 2$. We may assume that one of the components of $S-a_{m}$ is homeomorphic to a Riemann surface $S^{\prime}$ and the mapping $\pi_{1 \ldots m-1}$ : $\left(z_{1}, \cdots, z_{m}\right) \rightarrow\left(z_{1}, \cdots, z_{m-1}\right)$ is a surjection from $T(S)$ to $T\left(S^{\prime}\right)$. If follows that $T\left(S^{\prime}\right)=\pi_{1 \ldots m-1}(T(S))=U_{1} \times U_{2}^{\prime}$, where $U_{2}$ is an open subset of $U^{m-k-1}$ corresponding to the last $m-k-1$ coordinates $z_{k+1}, \cdots, z_{m-k-1}$. Since $m-k-1 \geqq 1$, this is a contradiction.

\section{$\S 5$. Parabolic transformations with invariant dises}

Related to the modular group, there is a problem: what kind of modular transformations leave some Teichmüller discs invariant? It is known that each hyperbolic transformation has an invariant Teichmüller disc (Bers [2]) and each pseudo-hyperbolic transformation has none. With respect to parabolic transformations it is also known (Marden-Masur [7]) that a product of Dehn twists about an admissible set of curves leaves a Teichmüller disc invariant if the factors of the product have simultaneously positive orders or negative orders. The converse of this fact, which was suggested to the first author by Earle's talk at the conference in Helsinki 1987 (cf. Earle-Sipe [3], Kra [4]), is shown by making use of Maskit coordinates;

THEOREM 2. Let $\chi$ be a parabolic element of $\operatorname{Mod}(S)$ with an invariant Teichmüller disc $\boldsymbol{D}$, and let $m$ be an integer such that $\chi^{m}$ is induced by a product of Dehn twists about an admissible set of curves $\Sigma$ on $S$. Then the factors of the product are simultaneously of positive orders or are simultaneously of negative orders.

Proof. Let $\chi, m, \boldsymbol{D}$ and $\Sigma$ be as above. Adding some Jordan curves to $\Sigma$, we get a maximal partition of $S$. We shall employ the Maskit coordinates of $T(S)$ defined by them. Then there exist $3 p-3+n$ integers $m_{1}, \cdots, m_{3 p-3+n}$ such that

$$
\begin{aligned}
\chi^{m}\left(z_{1}, \cdots, z_{3 p-3+n}\right)= & \left(z_{1}+m_{1}, \cdots, z_{3 p-3+n}+m_{3 p-3+n}\right) \\
& \left(z_{1}, \cdots, z_{3 p-3+n}\right) \in T(S)
\end{aligned}
$$

It is sufficient to prove all $m_{k}$ are simultaneously non-negative or non-positive.

Suppose that some of them, say $m_{1}$ is negative, and another, say $m_{2}$ is positive. Since $\boldsymbol{D}$ is a Teichmüller disk, there exists a biholomorphic mapping $\Psi=$ $\left(\psi_{1}, \cdots, \phi_{3 p-3+n}\right)$ of $U$ onto $\boldsymbol{D}$. As $\chi^{m}$ also leaves $\boldsymbol{D}$ invariant, $\Psi^{-1} \circ \chi^{m} \circ \Psi$ is an element of $P S L(2, \boldsymbol{R})$, and is parabolic since $\Psi$ is isometric with respect to the hyperbolic distance on $U$ and the Teichmüller distance on $T(S)$ (cf. Kra [4] p. 
267). Composing some element of $P S L(2, \boldsymbol{R})$ with $\Psi$, we may assume that

$$
\begin{aligned}
\Psi^{-1} \circ \chi^{m} \odot \Psi(z) & =T(z), \quad \text { or } \\
& =T^{-1}(z) \quad \text { on } U,
\end{aligned}
$$

where $T(z)=z+1$. In the first case, namely when $\chi^{m} \circ \Psi(z)=\Psi \circ T(z)$, we have

$$
\phi_{1}(z)+m_{1}=\phi_{1}(z+1)
$$

This implies that the holomorphic mapping $\phi_{1}$ of $U$ into $U$ is projected to a holomorphic mapping $f$ of $U /\langle T\rangle$ into $U /\langle T\rangle . \quad U /\langle T\rangle$ is identified with the punctured disc $D^{*}=\{0<|z|<1\}$. The origin is a removable singularity of $f$, and $f(0)=0$. In fact, by the Lindelöf's theorem and the above formula $\left(^{*}\right), \phi_{1}$ has a horospherical limit $\infty$ at $\infty$. Hence a closed curve around the origin is mapped to a closed curve around the origin. Considering $\left(^{*}\right)$, those with winding number one is mapped to those with winding number $m_{1}$. Since $f$ is orientation preserving, $m_{1}$ is non-negative. This contradicts the assumption.

In the latter case, a similar argument yields a contadiction.

\section{REFERENCES}

[1] Bers, L., On bounderies of Teichmüller spaces and on kleinian groups: I, Ann. of Math. (2) 91 (1970), 570-600.

[2] BERs, L., An extremal problem for quasiconformal mappings and a theorem by Thurston, Acta Math. 141 (1978), 73-98.

[3] EARle, C. J. AND SiPe, P.L., Families of Riemann surfaces over the punctured disk, to appear.

[4] KRA, I., On the Nielsen-Thurston-Bers type of some self-maps of Riemann surfaces, Acta Math. 146 (1981), 231-270.

[5] KRA, I., Canonical mappings between Teichmüller spaces, Bull. Amer. Math. Soc. 4 (1981), 143-179.

[6] KRA, I., Non-variational global coordinates for Teichmüller spaces, in "Holomorphic Functions and Moduli II", Springer-Verlag (1988), 221-249.

[7] MARDEN, A. AND MASUR, H., A foliation of Teichmüller space by twist invariant discs, Math. Scand. 36 (1975), 221-228.

[8] MaskiT, B., On bounderies of Teichmüller spaces and on Kleinian groups: II, Ann. of Math. 91 (1970), 607-639.

[9] Maskit, B., Moduli of marked Riemann surfaces, Bull. Amer. Math. Soc. 80 (1974), 773-777.

[10] Royden, H.L., Automorphisms and isometries of Teichmüller space, in "Advances in the Theory of Riemann Surfaces", Ann. of Math. Studies 66, Princeton Univ. Press (1971), 369-383.

[11] SHIGA, H., Remarks on holomorphic families of Riemann surfaces, Tôhoku Math. J. 38 (1986), 539-549. 
